\title{
Morphological and Biochemical Features of 'Atypical' Mycobacteria
}

\author{
BY ANNA CSILLAG \\ Medical Research Council Unit for Research on Drug Sensitivity in Tuberculosis, \\ Postgraduate Medical School of London, Ducane Road, London, W. 12
}

(Received 22 August 1960)

\begin{abstract}
SUMMARY
Morphological and biochemical features of 42 strains of 'atypical' mycobacteria and one strain of Mycobacterium tuberculosis were studied. Of the 42 atypical mycobacteria, 16 were originally classified in Runyon's group $I, 4$ in group II, 19 in group III, and 3 in group IV. The characteristics studied were bacillary morphology and staining properties on Kirschner and LöwensteinJensen media; colonial morphology on 7H-10 agar medium; pigmentation in the dark and after exposure to light; rate of growth and temperature requirements, with different methods of inoculation; growth on blood and nutrient agar plates, and in gelatin stabs; catalase activity on drug-free and on isoniazid-containing media; nicotinic acid (niacin) production. The sensitivity of the majority of the strains to 6 chemotherapeutic drugs was tested. The niacin test proved to be the most useful method for distinguishing the atypical mycobacteria from $M$. tuberculosis. In identifying strains of group $I$, their ability to produce yellow pigment after exposure to light was of most value, and their colonial morphology and their periodic acidSchiff staining were also helpful. Strains of group II were identified by their ability to form yellow pigment in the dark, by their periodic acid-Schiff staining and by their colonial morphology. Strains of group III were identified by their rate of growth and buff pigmentation. Exceptionally a yellow pigment was formed, and such strains were identified principally by their colonial morphology and periodic acid-Schiff staining. Among the 19 strains classified originally as group III, 3 were reclassified into group IV. Strains of group IV were identified by their ability to grow on blood and nutrient agar plates within 3 days and in gelatin stab cultures within 2 weeks.
\end{abstract}

\section{INTRODUCTION}

In the last few years several publications have appeared dealing with human diseases caused by 'atypical' mycobacteria (Wood, Buhler \& Pollak, 1956; Nassau \& Hamilton, 1957; Beck, 1959; Lewis et al. 1959; Engbaek, Magnusson \& Nielsen 1959). These authors, and others mentioned by Nassau \& Hamilton (1957), stated that the diseases caused by atypical mycobacteria and by Mycobacterium tuberculosis are closely similar. Thus the correct diagnosis and treatment depends upon the precise identification of the causative agent. The identification of an infection by $M$. tuberculosis in routine diagnostic work often depends on the morphology and the staining characteristics of the bacilli as seen in smears made from clinical specimens and sometimes from cultures, together with the colonial morphology on Löwen- 
stein-Jensen medium. These procedures are liable to considerable misinterpretation. The bacillary morphology and the degree of acid-fastness of $M$. tuberculosis and atypical mycobacteria may be similar. Colonies of $\boldsymbol{M}$. tuberculosis on LöwensteinJensen medium may closely resemble those of some other strains of mycobacteria. Several morphological and biochemical tests (reviewed by Freerksen, 1960) have been suggested as additional aids for differentiating atypical mycobacteria from M. tuberculosis, such as microcolonial structure (Engbaek, 1952), cord formation (Middlebrook, Dubos \& Pierce, 1947; Yegian \& Budd, 1953), pigmentation of colonies (Tarshis \& Frisch, 1952; Timpe \& Runyon, 1954), the neutral red test (Dubos \& Middlebrook, 1948; Hauduroy \& Posternak, 1949), catalase activity (Middlebrook, 1954) and nicotinic acid (niacin) production (Konno, 1956). No single test has been found to be completely satisfactory for this purpose. Runyon $(1959 a, b)$ proposed a classification of the atypical mycobacteria based mainly on pigmentation of colonies and speed of growth. The purpose of the present investigation was to examine the morphological characteristics and some of the biochemical properties of a series of strains of atypical mycobacteria, classified into Runyon's four groups, and to see whether these characteristics are of value in differentiating these strains from $M$. tuberculosis. All of the strains used were of human origin.

\section{Strains}

\section{METHODS}

Mycobacterium tuberculosis, 1 strain, H37 Rv. Atypical mycobacteria classified according to Runyon (1959a): group I, photochromogens (16 strains); group II, scotochromogens (4 strains); group III, Battey type (19 strains); group IV, rapidly growing (8 strains). The origin of the strains is set out in Table 1. All were obtained from human sputum or resected lung. The 18 British strains were obtained from 13 patients who had been examined in various British chest clinics and hospitals. Of these, were described by Selkon \& Mitchison (1959). All 29 strains from the United States of America were kindly sent by Dr E. H. Runyon, Veterans Administration Hospital, Salt Lake City, Utah, U.S.A.

Table 1. The mycobacterial strains examined

\begin{tabular}{|c|c|c|c|c|c|}
\hline \multirow[b]{2}{*}{ Group } & \multirow{2}{*}{$\begin{array}{l}\text { No. of } \\
\text { strains } \\
\text { tested }\end{array}$} & \multicolumn{2}{|c|}{ Great Britain } & \multicolumn{2}{|c|}{ United States of America } \\
\hline & & $\begin{array}{l}\text { No. of } \\
\text { strains }\end{array}$ & $\begin{array}{c}\text { Strain } \\
\text { no. }\end{array}$ & $\begin{array}{l}\text { No. of } \\
\text { strains }\end{array}$ & $\begin{array}{c}\text { Strain } \\
\text { no. }\end{array}$ \\
\hline I. Photochromogen & 16 & 10 & $\begin{array}{l}\text { 0523, 0735, } \\
\text { 1438, 1526S, } \\
\text { 1526R, M1159, } \\
\text { M1212, M1246, } \\
\text { M1247, A725 }\end{array}$ & 6 & $\begin{array}{l}15,260,265 \\
266,353,440\end{array}$ \\
\hline II. Scotochromogen & 4 & $\mathbf{2}$ & 0112, M1177 & 2 & 50,251 \\
\hline III. Battey type & 19 & $\mathbf{1}$ & M1208 & 18 & $\begin{array}{r}160,170,171 C, \\
223,225,277, \\
248,470,477, \\
487,509,513, \\
514,520,585, \\
\text { P7, P23, P25 }\end{array}$ \\
\hline IV. Rapidly growing & $\mathbf{3}$ & - & - & $\mathbf{3}$ & $880,481,518$ \\
\hline
\end{tabular}


Examination of cultures in Kirschner medium. Kirschner medium (Mackie \& McCartney, 1956) was inoculated from Löwenstein-Jensen cultures in screw-capped bottles, which were 3 weeks or less old, and which had not been opened since inoculation. Care was taken not to transfer lumps of bacteria to the Kirschner medium. After incubation for 14 days at $87^{\circ}$, films were made and they were then dried under an ultraviolet lamp for $\mathbf{3 0}$ min., placed in methanol for $5 \mathrm{~min}$., and rinsed in water. Ziehl-Neelsen staining was performed in the usual way, with 5 min. decolorization with acid-alcohol ( $\mathrm{NaCl} 20 \mathrm{~g}$., $\mathrm{HCl}$ conc. $20 \mathrm{ml} ., 7^{\circ}$ O.P. Spirit $1500 \mathrm{ml}$., distilled water $400 \mathrm{ml}$.) and counterstaining with Löfler's methylene blue for 5-15 min. Periodic acid-Schiff staining was performed as described by Csillag (1960).

Examination of cultures on Lörwenstein-Jensen medium. Cultures were grown on Löwenstein-Jensen medium for $\mathbf{B}$ weeks at $37^{\circ}$. Smears, prepared and fixed as described above, were stained by the Ziehl-Neelsen and the Ziehl-Heidenhain (Uyeda, 1955) methods.

Colonial morphology. The morphology of colonies was examined on plates of oleic acid + albumin agar medium (7H-10 medium; Cohn, Middlebrook \& Russell, 1959). The plates were inoculated from a 4 -week growth on the same medium and incubated for $8-4$ weeks at $37^{\circ}$ in a hot room where they were occasionally exposed to artificial illumination. The colonies were formolized, and observed with a plate microscope ( $\times 10$ magnification).

Pigmentation folloroing incubation in the dark or after exposure to light. Two Löwenstein-Jensen medium slopes were inoculated with each strain. One of these was placed in a sealed box and incubated at $87^{\circ}$ in the dark. The other slope was also incubated at $37^{\circ}$ and, when growth first appeared, it was exposed to diffuse daylight for $1 \mathrm{hr}$., re-incubated for $24 \mathrm{hr}$. and compared for its pigmentation with the slope incubated in the dark. When only minor differences in pigmentation were found between the two slopes, the procedure was repeated on another batch of medium. Those strains which produced yellow or orange pigment when grown in the dark were examined in the same manner, but when growing on 7H-10 medium slopes.

Temperature requirements and rate of growth. The rate of growth at $37^{\circ}$ and at $22^{\circ}$ was studied on Löwenstein-Jensen medium slopes which had been inoculated with a loop containing a small amount of growth from a 3- to 4-week culture on the same medium ('clumped inoculum' method). With the photochromogens, the rate of growth at the same temperatures was also studied on the same medium which had been inoculated with a $8 \mathrm{~mm}$. loopful of a suspension prepared by shaking about $2 \mathrm{mg}$. (moist weight) of bacillary mass with $0 \cdot 4 \mathrm{ml}$. distilled water in a screw-capped bottle containing glass beads ('dispersed inoculum' method). Cultures were examined at daily intervals (except on Sundays) for 6 weeks.

Growth on blood and nutrient agar. Nutrient agar plates with or without $5 \%(\mathrm{v} / \mathrm{v})$ horse blood were inoculated from a 3- to 4-week growth on $7 \mathrm{H}-10$ agar plates. The cultures were incubated at $37^{\circ}$ for 3 days.

Growoth in gelatin stabs. Nutrient gelatin stab cultures were inoculated with a straight wire from a 3- to 4-week growth on 7H-10 medium plates. The cultures were incubated at $22^{\circ}$ for 6 weeks.

The nicotinic acid (niacin) test. Tests for nicotinic acid were carried out as described by Gilani \& Selkon (1958), after incubation for 6 weeks. 
Catalase activity. Catalase activity (Middlebrook, 1954; Selkon \& Mitchison, 1959) was studied on growth on drug-free Löwenstein-Jensen slopes and on slopes containing isoniazid in the sensitivity tests. In studying the catalase activity of isoniazid-resistant variants, Löwenstein-Jensen slopes containing $50 \mu \mathrm{g}$. isoniazid/ ml. were inoculated with a large amount of bacillary growth, incubated for 4 weeks at $87^{\circ}$ and the resulting growth tested.

Sensitivity tests. Sensitivity tests to isoniazid, $p$-aminosalicylic acid, streptomycin and $p$-acetamidobenzaldehyde thiosemicarbazone (thiacetazone) were performed as described by Selkon \& Mitchison(1959). The sensitivity to 2-ethylisothionicotinamide (thioamide) and cycloserine was tested in a similar manner, with twofold serial drug dilutions in Löwenstein-Jensen medium. The results of a single test on each strain are reported; unexpected results were not repeated.

\section{RESULTS}

\section{Bacillary morphology and staining}

The strains were divided into types on the basis of their bacillary morphology (' $B M$ ') type and staining properties, as follows.

\section{Group I}

BM type I a (15 strains, all except no. 1526R). Majority of organisms longer than those of Mycobacterium tuberculosis $\mathrm{H3r} \mathbf{R v}$, and strongly beaded. Tendency to formation of loose cords present, especially in Kirschner medium. Not acid-fast organisms only exceptionally seen with both staining methods, with $5 \mathrm{~min}$. or 15 min. counterstaining. Periodic acid-Schiff staining positive.

BM type Ib (1 strain, no. 1526 R). As above, but no tendency to cord formation, either in Kirschner or on Löwenstein-Jensen medium.

\section{Group II}

BM type II (4 strains). Size of organisms variable. Majority of organisms beaded. Cord-formation not observed in either medium. Not acid-fast organisms only exceptionally seen with both staining methods. Periodic acid-Schiff staining positive.

\section{Group III}

BM type III a (10 strains; all nos. except those listed as types IIIb-IIId below). Size of organisms nearly uniform and shorter than those of Mycobacterium tuberculosis H87 Rv. Beaded or uniform staining. Tendency to cord formation not observed. Not acid-fast organisms rarely seen in growth on Kirschner medium. More not acidfast organisms seen in growth on Löwenstein-Jensen medium, especially when stained either by the Ziehl-Neelsen method, with 15 min. counterstaining, or by the Ziehl-Heidenhain method. Periodic acid-Schiff staining negative.

BM type III b (7 strains, nos. P7, P23, 170, 223, 277, 487, M 1208). As for type IIIa, but organisms not uniform in size; filaments, rods and coccoid forms being seen in the same smear. Nearly half of the organisms not acid-fast in growth on Löwenstein-Jensen medium, with all staining methods. Majority of organisms not acidfast in Kirschner medium. Periodic acid-Schiff staining negative.

BM type III $c$ (1 strain: no 171). As for type IIIb, but, after 8 preliminary serial subcultures at monthly intervals on Löwenstein-Jensen medium, exclusively not 
acid-fast organisms seen in both media. Organisms of types IIIa, IIIb and IIId retained acid-fastness during similar passages. Periodic acid-Schiff staining negative.

BM type IIId (1 strain: no. 160). As for type III a, but tight cords present in growth from Kirschner medium. Periodic acid-Schiff staining negative.

\section{Group IV}

BM type IV (3 strains). Size of organisms similar to those of Mycobacterium tuberculosis Ha7 Rv. Staining uniform. Tight cords present. Not acid-fast organisms only exceptionally seen in both media, with both staining methods. Periodic acidSchiff staining negative.

Mycobacterium tuberculosis (H37 Rv). Size of organisms typical for M. tuberculosis. Staining uniform or beaded. Tight cords present. Not acid-fast elements only exceptionally seen in both media, with both staining methods. Periodic acid-Schiff staining negative.

\section{Colonial morphology}

The strains were divided into types on the basis of their colonial morphology ('CM' types) on 7H-10 medium, as follows.

\section{Group I}

CM type Ia (13 strains, all nos., except those listed as type Ib). Circular, differences in size slight, low convex, finely granular surface, slightly crenated edge, central area of opacity, pigmented buff, butyrous.

CM type Ib (3 strains, nos. 440, 0735, 1526R). Circular, differences in size slight, raised, coarsely granular surface, crenated edge, some colonies umbonate, heaping between adjacent colonies sometimes seen, pigmented buff, friable, difficult to emulsify.

\section{Group II}

CM type II (4 strains). Circular, nearly uniform in size, high, convex, smooth surface, uniform structure, entire edge, pigmented yellow, butyrous.

\section{Group III}

CM type IIIa (11 strains: all nos. except those listed as types IIIb-IIIe below). Circular, size variable, domed, smooth surface, uniform structure, entire or slightly crenated edge, pigmented buff, butyrous.

CM type III ( 2 strains, nos, 514, 520). As for type III a, but pigmented yellow.

CM type IIIc (1 strain, no. 513). As for type IIIa, but colonies irregular in shape.

CM type IIId (1 strain; no. 470). As for type III a, but size uniform and central area of opacity.

CM type IIIe (4 strains: nos. 160, 170, 225, 509). Circular, size very variable, effuse, umbonate, finely granular surface, entire or crenated edge, pigmented buff, friable.

\section{Group IV}

CM type IV (3 strains). Circular, size variable, low convex, coarsely granular surface, crenated edge, some colonies in each strain with centre umbonate, heaping between adjacent colonies seen, pigmented buff, friable. 
Mycobacterium tuberculosis. Circular, size slightly variable, low convex, coarsely granular surface, crenated edge, centre umbonate, heaping between adjacent colonies seen, pigmented buff, friable.

\section{Pigmentation}

Pigmentation was observed on colonies grown systematically in the dark or exposed to light, with the following results.

Group I. All strains buff, when grown on Löwenstein-Jensen medium in the dark, yellow after $1 \mathrm{hr}$. exposure to light. One strain (no. 0735) pigmented yellow only after two exposures of $8 \mathrm{hr}$. to light.

Group II. Pigmented deep yellow in the dark and in the light on LöwensteinJensen and on 7 H-10 medium.

Group III. Strains 514, 520, M1208 pigmented deep yellow in the dark and in the light on Löwenstein-Jensen and 7H-10 media. All remaining strains pigmented buff in the dark and in the light.

Group IV and Mycobacterium tuberculosis. Pigmented buff in the dark and in the light.

\section{Temperature requirements and rate of growth}

All of the atypical mycobacteria grew at $22^{\circ}$ within 6 weeks when the cultures were inoculated by the clumped inoculum method. Among strains of Mycobacterium tuberculosis, H37 Rv did not grow at this temperature; in further experiments with other strains growth occurred in a small proportion of them. Among the 16 group I strains whose cultures were inoculated by the dispersed inoculum method, three did not grow at $22^{\circ}$ (nos. A 725, M 1247, 0523). Considerable differences were found in the rate of growth at $37^{\circ}$, depending upon the inoculum used. Growth was more rapid with a clumped inoculum than with a dispersed inoculum and, with the group III strains, growth occurred 3-4 days earlier when the slopes were inoculated from 6-week cultures than from 2- to 3-week cultures. Whichever inoculum was used, group IV strains grew more rapidly than the remaining strains of atypical mycobacteria, and these grew slightly more rapidly (1-4 days quicker) than M. tuberculosis $\mathbf{H a r} \mathbf{R v}$.

\section{Growth on blood and nutrient agar}

Strains of group I and group II did not grow within 5 days on blood agar and nutrient agar plates. Of the 19 group III strains, 16 did not grow on these media in this period, but 3 strains (nos. P25, 487, 171 C) yielded growth within 3 days. All group IV strains grew within 3 days. Mycobacterium tuberculosis $\mathbf{H 3 7} \mathbf{R v}$ did not grow.

\section{Growth in gelatin stabs}

Group I strains, group II strains, Mycobacterium tuberculosis $\mathrm{H} 37 \mathrm{Rv}$ and those group III strains which did not grow on blood and nutrient agar, did not grow in gelatin stabs. The 3 group III strains, which yielded growth on blood and nutrient agar, grew in gelatin stabs within 2 weeks. These strains formed colonies on the top and a filiform growth in the stab; liquefaction was not observed. All group IV strains grew within 2 weeks, forming colonies on the tops and a filiform growth in the stabs, and did not liquefy the medium. 
The nicotinic acid test

All strains of atypical mycobacteria yielded a negative nicotinic acid test, while Mycobacterium tuberculosis $\mathbf{H B z} \mathbf{R v}$ yielded a positive result.

\section{Catalase activity}

The results of catalase tests are shown in Table 2. All group I, group II and group IV strains showed catalase activity greater than with Mycobacterium tuberculosis $\mathbf{H B 7} \mathrm{Rv}$ when grown on drug-free medium and on medium containing isoniazid, as did their isoniazid-resistant variants. Group III strains showed a variable pattern of catalase activity, some strains having activities similar to those of the remaining atypical strains and others yielding a pattern more closely resembling that of Mycobacterium tuberculosis $\mathbf{H 3 7} \mathbf{R v}$.

Table 2. Catalase activity of mycobacteria

\begin{tabular}{|c|c|c|c|c|}
\hline & & & Catalase activity* & \\
\hline & & Origin & strain & \\
\hline Group & Strain no. & $\begin{array}{l}\text { Growth on drug } \\
\text { free medium }\end{array}$ & $\begin{array}{l}\text { Growth on } \\
\text { medium containing } \\
\text { highest isoniazid } \\
\text { concentration } \\
\text { allowing growth }\end{array}$ & $\begin{array}{c}\text { Variant resistant } \\
\text { to } 50 \mu \mathrm{g} . \\
\text { isoniazid/ml. }\end{array}$ \\
\hline $\mathbf{I}$ & All 16 & +++ & $++t$ & $++t$ \\
\hline II & All 4 & +++ & +++ & $+t+$ \\
\hline III & $\begin{array}{l}171 \mathrm{C}, 487,513, \\
514,520, \text { P7, } \\
\text { P25 }\end{array}$ & +++ & +++ & $++t$ \\
\hline & $\begin{array}{l}\text { 160, 225, 470, } \\
\text { P23 }\end{array}$ & +++ & ++ & ++ \\
\hline & $\begin{array}{l}248,277,609 \\
585\end{array}$ & ++ & $+t$ & ++ \\
\hline & $\begin{array}{l}170,477 \\
223, \text { M1208 }\end{array}$ & $\begin{array}{l}++ \\
++\end{array}$ & $\begin{array}{c}++ \\
+\end{array}$ & $\begin{array}{l}0 \\
+\end{array}$ \\
\hline IV & All 3 & +++ & +++ & +++ \\
\hline M. tuberculosis & HarRv & $+t$ & $\mathbf{0}$ & $\mathbf{0}$ \\
\hline
\end{tabular}

- Catalase activity scored by number of + signs.

Catalase activity more marked than that of strain Mycobacterium tuberculosis $\mathbf{H B r} \mathbf{R v},+++$; equal to that of strain $\mathbf{H 3 7} \mathrm{Rv},++$; present, but less than that of strain $\mathbf{H B r} \mathbf{R v}$, + ; no activity, 0 .

\section{Sensitivity tests}

The results of the sensitivity tests are set out in Tables 3 and 4. Classifications as sensitive, doubtfully resistant, and resistant are based on experience with strains of Mycobacterium tuberculosis. All of the strains of atypical mycobacteria were resistant to isoniazid and all were resistant to $p$-aminosalicylic acid, with three exceptions (group I, strains nos. 0523, 0735 and 260). All group IV strains were resistant to streptomycin, but about one third of the remainder were sensitive. All of the group I strains were sensitive or doubtfully resistant to cycloserine. Of the 22 group II and group III strains tested, one in each group (nos. 0112, 170) was resistant to cycloserine, as were all of the group IV strains. All of the group I and 


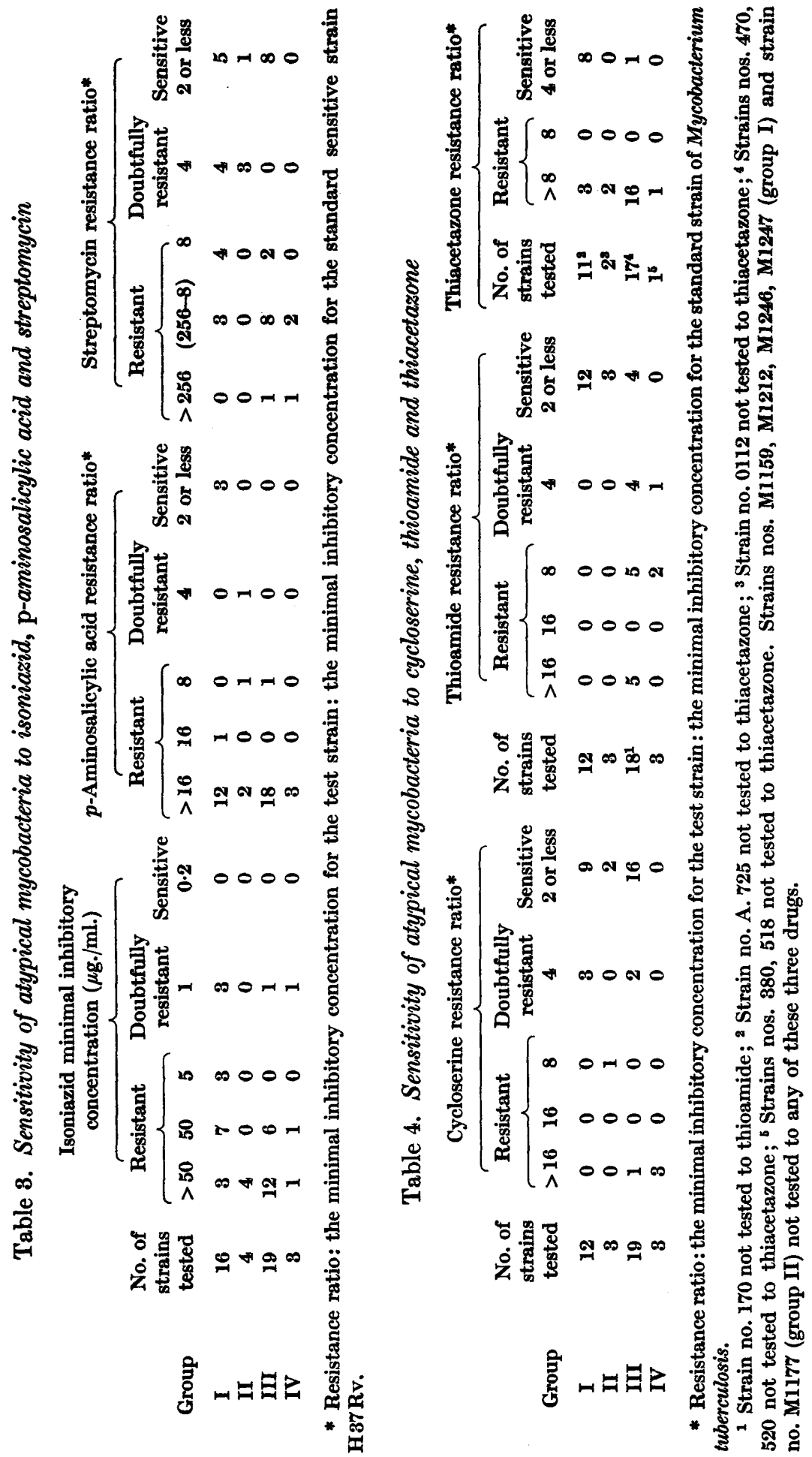


group II strains were sensitive to thioamide and the remaining groups showed a variable pattern of sensitivity. Of the 11 group I strains tested, 8 were sensitive to thiacetazone whereas all except one of the remaining strains (no. 514) were resistant. These sensitivity tests were not repeated systematically and, from further experience, a slightly different pattern of resistance, particularly to $p$-aminosalicylic acid and thiacetazone, might have been encountered if several tests had been set up on each strain.

\section{Characteristics of the different groups}

Those features of the strains belonging to the four groups which were found to be most consistent and characteristic of the groups, are given below.

Group $I$. Long, beaded, strongly acid-fast, periodic acid-Schiff positive. Tendency to cord formation. Colonies of the smooth strains (CM type Ia) have central areas of opacity. Colonies of the rough strains (CM type Ib) similar to those of Mycobacterium tuberculosis, strain H37Rv. Yellow pigmentation only after exposure to daylight. No growth on blood and nutrient agar at $37^{\circ}$ within 3 days, and no growth in gelatin stabs at $22^{\circ}$ within 6 weeks. Nicotinic acid test negative. Catalase activity strongly positive even when grown on isoniazid-containing medium. Resistant to isoniazid; sensitive to thioamide; sensitive or doubtfully resistant to cycloserine; often sensitive to thiacetazone.

Group II. Beaded, strongly acid-fast, period acid-Schiff positive organisms of variable length. No tendency to cord formation. Colonies smooth, with entire edges. Pigmented deep yellow when grown in the dark, as well as after exposure to light. No growth on blood and nutrient agar at $37^{\circ}$ within 3 days and no growth in gelatin stabs at $22^{\circ}$ within 6 weeks. Nicotinic acid negative. Catalase activity strongly positive, even when grown on isoniazid-containing medium. Resistant to isoniazid and thiacetazone; sensitive to thioamide; sensitive or doubtfully resistant to streptomycin.

Group III. Organisms either uniformly shorter than those of Mycobacterium tuberculosis H37 Rv or pleomorphic. Staining either uniform or beaded. Either all organisms acid-fast or many not acid-fast. No tendency to cord formation (tight cord formed by one strain). Periodic acid-Schiff negative. Colonies of smooth strains (CM type III a-IIId) variable in size, with entire edges and uniform structure. (One strain with central area of opacity.) Colonies of rough strains (CM type IIIe) similar to those of $M$. tuberculosis strain $\mathbf{H 3 7} \mathbf{R v}$, but smaller and more variable in size. Colonies of 16 strains pigmented buff, but colonies of the remaining 3 strains pigmented yellow. No growth on blood and nutrient agar at $37^{\circ}$ within 3 days, and no growth in gelatin stabs within 6 weeks (3 exceptions). Catalase activity variable, often diminished when grown on isoniazid-containing slopes. Resistant to isoniazid and $p$-aminosalicylic acid; sensitive or doubtfully resistant to cycloserine (1 exception).

Group IV. Bacillary morphology, staining properties (including acid-fastness and periodic acid-Schiff staining), morphology of colonies and pigmentation the same as those of Mycobacterium tuberculosis, strain $\mathrm{H37} \mathbf{R v}$. Growth on blood and nutrient agar at $37^{\circ}$ within 3 days, and growth in gelatin stabs at $22^{\circ}$ within 6 weeks. Nicotinic acid test negative. Catalase activity strongly positive even when grown on isoniazid-containing medium. Resistant to isoniazid, $p$-aminosalicylic acid, streptomycin and cycloserine; resistant or doubtfully resistant to thioamide. 


\section{DISCUSSION}

Two main problems arise during the course of identifying mycobacteria obtained from material of human origin: (1) the differentiation of Mycobacterium tuberculosis from the atypical mycobacteria, and (2) their classification. In these experiments, the most useful method for differentiating $M$. tuberculosis from the atypical mycobacteria was found to be the nicotinic acid test. All of the strains of atypical mycobacteria yielded a negative result, whereas strain $M$. tuberculosis $\mathrm{H37} \mathrm{Rv}$ gave a positive result. Using the same technique, Gilani \& Selkon (1958) found that all of 13 human strains of $M$. tuberculosis yielded a positive nicotinic acid test. Runyon, Selin \& Harris (1959), who used a different technique, reported their own findings and those of other workers, and found that the nicotinic acid test was uniformly negative for atypical mycobacteria and uniformly positive for human $M$. tuberculosis. The main disadvantage of the nicotinic acid test is that bovine strains of $M$. tuberculosis yield a negative result, so that strains of mycobacteria which yield such a result must be further characterized, for example by guinea-pig inoculation. Growth at $\mathbf{2 2}^{\circ}$ was not reliable as a means of differentiating $M$. tuberculosis from atypical mycobacteria since with the clumped inoculum occasional strains of $\boldsymbol{M}$. tuberculosis yielded growth, and with the dispersed inoculum some of the atypical mycobacteria tested did not grow. Growth at $25^{\circ}$ may be more satisfactory (Marks \& Trollope, 1960). The following characteristics were also of value in identifying atypical strains : long, beaded organisms; weakly acid-fast; lack of cord formation; bacillary pleomorphism; periodic acid-Schiff positivity; smoothness of colonies; yellow pigmentation of colonies; growth on blood and nutrient agar within 3 days; growth in gelatin stabs cultures; high catalase activity on medium containing isoniazid and a characteristic sensitivity pattern. No one of these characteristics was invariably shown by all of the atypical strains. However, when several of these characters were examined, all of the atypical strains were distinguishable from M. tuberculosis.

The following characteristics were found to be of most value in classifying the atypical mycobacteria into the groups proposed by Runyon (1959a). Group I: formation of yellow pigment after exposure to light for $1 \mathrm{hr}$. was described as the essential characteristic of strains in this group. However, one of our strains required a longer period of exposure, but it was still included with other strains in this group because of the similarity of their behaviour in other respects. Strains with smooth colonies (CM type Ia) could usually be classified by their colonial morphology, but rough colonies (CM type Ib) could be confused with those of Mycobacterium tuberculosis and of group IV strains. Positive periodic acid-Schiff staining was an invariable feature in this group but was also found in group II strains. Group II : formation of yellow or orange pigment in the dark (Runyon, 1959a) was invariably found with all strains, but also occurred with 3 of the group III strains. Positive periodic acidSchiff staining was shown by all strains in this group, and also by those in group $I$. Colonial morphology was also of value. Group III: among the 19 strains classified in this group, 3 yielded yellow pigmentation both in the dark and in the light, and on both Löwenstein-Jensen and 7 H-10 media. Nevertheless, they resembled the remaining strains in this group in other characteristics and should be retained in it. A further 3 strains grew on nutrient agar and Löwenstein-Jensen medium within 
8 days and they also grew in gelatin stab cultures. It would therefore be reasonable to transfer these 3 strains to group IV. With the remaining strains classified in group III, diversity of all of the characteristics studied was noted, and this group cannot be considered as homogeneous. Group IV: Runyon did not describe in detail the specific conditions necessary to identify these strains as rapidly-growing ones. In the present work the speed of growth of the strains in all of the groups on Löwenstein-Jensen medium was dependent on the size and type of the inoculum, so that it was not always easy to distinguish group IV strains from the remainder. The ability to grow on blood and nutrient agar plates within $\mathbf{3}$ days was found only with group IV strains and with the three group III strains mentioned above. The superiority of the test on blood or nutrient agar may be due not only to differences in growth rate, but also the more satisfactory nature of these media for group IV strains. The ability to grow in gelatin stab cultures within 6 weeks was also of value for characterizing these strains. Further experience with 32 strains of saprophytic mycobacteria confirms the value of blood and nutrient agar and of gelatin stab cultures in their identification as group IV strains.

I wish to thank Dr D. A. Mitchison for his many helpful suggestions. Thanks are also due to Miss Janet Lloyd for her excellent technical assistance.

\section{REFERENCES}

BeCK, F. (1959). Pulmonary disease due to atypical tubercle bacilli. Amer. Rev. resp. Dis. 80, 788.

Csmung, A. (1960). Periodic acid-Schiff (PAS) staining of 'atypical' mycobacteria and tubercle bacilli. Tubercle, Lond. 41, 63.

Dubos, R. J. \& Mmprabroox, G. (1948). Cytochemical reaction of virulent tubercle bacilli. Amer. Rev. Tuberc. 58, 689.

EngBagk, H. C. (1952). Growth of Mycobacterium tuberculosis determined by direct agar microscopy. Acta path. microbiol. scand. 31, 369.

Engbagk, H. C., Magnusgon, M. \& Nmensen, J. A. (1959). A further case of lung disease caused by an atypical acid-fast organism. Acta Tuberc. scand. 37, 227.

Frakrksen, E. (1960). Die sogenannten atypischen Mycobacterien. Klin. Wschr. 38, 297.

Gruani, S. \& Skukon, J. B. (1958). The niacin test for differentiating human tubercle from other mycobacteria. Tubercle, Lond. 39, 396.

Gohn, M. L., Mmblembook, G. \& Russew, W. F. (1959). Combined drug treatment of tuberculosis. J. clin. Invest. 38, 1349.

Hauduroy, P. \& Postrarnak, Y. (1949). Sur une réaction permettent de distinguer les mycobactéries virulentes des mycobactéries avirulentes. C.R. Acad. Sci., Paris, $228,781$.

KonNo, K. (1956). New chemical method to differentiate human-type tubercle bacilli from other mycobacteria. Science, 124, 985.

Lewis, A. G., Dunbar, F. P., Lasche, E. M., Bond, J. O., Lerner, E. N., Wharton, D. J., HaRdy, A. V. \& Davies, R. (1959). Chronic pulmonary diseases due to atypical mycobacterial infections. Amer. Rev. resp. Dis. 80, 188.

Mackie, T. J. \& McCantiney, J. E. (1956). Handbook of Practical Bacteriology, 9th ed. p. 189. Edinburgh: Livingstone.

Marks, J. \& Troulope, D. R. (1960). A study of the 'Anonymous' mycobacteria. I. Introduction; colonial characteristics and morphology ; growth rates ; biochemical tests. Tubercle, Lond. 41, 51.

MmDLempook, G. (1954). Isoniazid resistance and catalase activity of tubercle bacilli. Amer. Rev. Tuberc. 69, 471. 
Midderenook, G., Dusos, R. J. Puknce, C. H. (1947). Virulence and morphological characteristics of mammalian tubercle bacilli. J. exp. Med. 86, 175.

Nassau, E. \& Humuton, G. M. (1967). Atypieal mycobacteria in human pulmonary disease. Tubercle, Lond. 38, 387.

RUNYoN, E. H. (1959a). Anonymous mycobacteria in pulmonary disease. Med. Clin. N. Amer. 43, 278.

RuNYON, E. H. (1959b). The recognition and characterization of pulmonary mycobacterial pathogens other than tubercle bacilli: review. Bull. int. Un. Tuberc. 29, 896.

Runyon, E. H., Serre, M. J. \& Harars, H. Ww. (1959). Distinguishing mycobacteria by the niacin test. Amer. Rev. Tuberc. 79, 663.

Seukon, J. B. \& Mrtchroon, D. A. (1959). Atypical mycobacteria and drug-resistant tubercle bacilli isolated during a survey of untreated patients with pulmonary tuberculosis. Tubercle, Lond. 40, 141.

Tarshis, M. B. \& Frisch, A. W. (1952). Chromogenic acid-fast bacilli from human sources. Amer. Rev. Tuberc. 65, 278.

Tmupe, A. \& Runyon, E. H. (1954). The relationship of 'atypical' acid-fast bacteria to human disease. J. Lab. clin. Med. 44, 202.

UYEDA, S. (1955). Contribution l'étude de la morphologie et du mode de développement du bacille tuberculeux. Rev. Tuberc., Paris, 19, 984.

Wood, L. E., Buhler, V. B. \& Polrak, A. (1956). Human infection with the 'yellow' acid-fast bacillus. Amer. Rev. Tuberc. 73, 917.

Yegran, D. \& Budd, V. (1958). Certain nonpathogenic mycobacteria. Amer. Rev. Tuberc. 68, 557. 